\title{
POSITIVE HERMITIAN CURVATURE FLOW ON COMPLEX 2-STEP NILPOTENT LIE GROUPS
}

\author{
MATTIA PUJIA
}

\begin{abstract}
We study the positive Hermitian curvature flow of left-invariant metrics on complex 2-step nilpotent Lie groups. In this setting we completely characterize the long-time behaviour of the flow, showing that normalized solutions to the flow subconverge to a non-flat algebraic soliton, in CheegerGromov topology. We also exhibit a uniqueness result for algebraic solitons on such Lie groups.
\end{abstract}

\section{INTRODUCTION}

In 2011, Streets and Tian introduced a new family of parabolic flows generalizing the Kähler-Ricci flow to the Hermitian setting [26]. More precisely, given a complex manifold $X$, any flow in the family evolves a Hermitian metric $g$ on $X$ via

$$
\partial_{t} g_{t}=-S\left(g_{t}\right)+Q\left(g_{t}\right), \quad g_{\left.t\right|_{0}}=g,
$$

where $S(g)$ is the second Chern-Ricci curvature tensor of $g$ on $X$ and $Q(g)$ is a $(1,1)$-symmetric tensor quadratic in the torsion of the Chern connection. The flows belonging to such a family are usually called Hermitian curvature flows (HCFs for short).

In [26], Streets and Tian chose the tensor $Q$ in order to obtain a gradient flow, stable near KählerEinstein metrics with non-positive scalar curvature and satisfying many other analytical properties; while, in the subsequent paper 25, $Q$ was chosen so that the pluriclosed condition was preserved $(\partial \bar{\partial} \omega=0)$ (see also [23, 24, 27, 28]). On the other hand, since the tensor $Q$ does not affect the parabolicity of the evolution equation (11), different choices of $Q$ can be performed in order to preserve other properties.

In [30, following Streets and Tian's approach, Ustinovskiy introduced a HCF preserving both the Griffiths-positivity and the dual Nakano-positivity of the tangent bundle. More precisely, given a compact Hermitian manifold $(X, g)$, Ustinovskiy considered the evolution equation

$$
\partial_{t} g_{t}=-S\left(g_{t}\right)-\widetilde{Q}\left(g_{t}\right), \quad g_{\left.t\right|_{0}}=g .
$$

Here, we denote by: $\nabla$ the Chern connection of $(X, g), \Omega$ the curvature tensor of $\nabla, S(g)$ the $(1,1)$ symmetric tensor

and $\widetilde{Q}(g)$ the tensor given by

$$
S_{i \bar{j}}=g^{k \bar{l}} \Omega_{k \bar{l} \bar{j}},
$$

where $T_{k m \bar{j}}:=g_{l \bar{j}} T_{k m}^{l}$ and $T_{k m}^{l}$ are the components of the torsion of $\nabla$. We will refer to (2) as to the positive Hermitian curvature flow $\left(\mathrm{HCF}_{+}\right.$for short) and we set

$$
K(g):=S(g)+\widetilde{Q}(g),
$$

for any Hermitian metric $g$ on $X$.

The aim of the present paper is to study the behaviour of the $\mathrm{HCF}_{+}$on complex nilpotent Lie groups when the initial metrics are left-invariant. Although in the non-compact case existence and uniqueness of left-invariant solutions to (2) are not always guaranteed, in our setting the invariance by biholomorphisms

Date: September 23, 2020.

2010 Mathematics Subject Classification. Primary 53C44; Secondary 53C15, 53C07, 53B15.

This work was supported by G.N.S.A.G.A. of I.N.d.A.M. 
MATTIA PUJIA

of the flow implies that (2) can be reduced to an ODE on the Lie algebra of the group. Hence, existence and uniqueness among left-invariant solutions follow from the standard ODE theory.

Our first result completely describes the long-time behaviour of the $\mathrm{HCF}_{+}$on a complex 2-step nilpotent Lie group.

Theorem A. Any left-invariant solution $g_{t}$ to the $H C F_{+}$on a complex 2-step nilpotent Lie group is immortal, and $(1+t)^{-1} g_{t}$ subconverges as $t \rightarrow \infty$ to a non-flat algebraic soliton $(\bar{N}, \bar{g})$, in the CheegerGromov topology.

By convergence in the Cheeger-Gromov topology we mean that: there exists a family of biholomorphisms $\varphi_{t}: \Omega_{t} \subset \bar{N} \rightarrow \varphi_{t}\left(\Omega_{t}\right) \subset N$ mapping the identity of $\bar{N}$ into the identity of $N$, such that the open sets $\left\{\Omega_{t}\right\}$ exhaust $\bar{N}$, and in addition $\varphi_{t}^{*} g_{t} \rightarrow \bar{g}$ as $t \rightarrow \infty$, uniformly over compact subsets in the $C^{\infty}$-topology.

Next we focus on soliton solutions to the flow. A Hermitian metric $g$ on a complex Lie group $G$ is a soliton to the $\mathrm{HCF}_{+}$if

$$
K(g)=c g+\mathcal{L}_{Z} g,
$$

for some $c \in \mathbb{R}$ and a complete holomorphic vector field $Z$. Here $\mathcal{L}$ denotes the Lie derivative. Notice that, soliton metrics are rather important in the study of the $\mathrm{HCF}_{+}$. Indeed, by the scale and biholomorphisms invariance of the $\mathrm{HCF}_{+}$-tensor, any initial metric $g$ satisfying (3) gives rise to a self-similar solution

$$
g_{t}=s(t) \varphi_{t}^{*}(g)
$$

to the $\mathrm{HCF}_{+}$, for some smooth scaling function $s(t)>0$ and a one-parameter family of biholomorphisms $\varphi_{t}: G \rightarrow G$. If furthermore $g$ is left-invariant and $\varphi_{t}$ may be chosen to be a family of Lie group automorphisms, then the soliton $g$ is said to be algebraic.

Our second result is about the uniqueness of algebraic solitons on complex 2-step nilpotent Lie groups.

Theorem B. Any complex 2-step nilpotent Lie group $N$ admits at most one algebraic $\mathrm{HCF}_{+}$-soliton up to homotheties. Moreover, any algebraic $\mathrm{HCF}_{+}$-soliton on $N$ is expanding (i.e. $c<0$ in (3)).

The proofs of our results are mainly based on the bracket flow technique, a powerful tool introduced by Lauret to study different geometric flows on homogeneous spaces (see e.g. [11), and on a moment map result which we prove in Section 2 .

We mention that recently many works on different HCFs on homogeneous spaces appeared. In 29] Ustinovskiy studied the $\mathrm{HCF}_{+}$on complex homogeneous manifold focusing on a distinguished class of non-homogeneous metrics, namely the class of induced metrics; while, the $\mathrm{HCF}_{+}$on $C$-spaces has been investigated by Panelli and Podestà in [12. Moreover, in [22] Stanfield studied the $\mathrm{HCF}_{+}$on almostabelian complex Lie groups.

The general behaviour of the 'original' HCF on complex unimodular Lie groups was studied by Lafuente, Vezzoni and the author in [6, where it is proved that any left-invariant solution to the flow is immortal and converges to an expanding algebraic soliton, which has to be unique up to homotheties. Moreover, in [19] it has been shown that expanding algebraic solitons on complex Lie groups lead to strong constrains on the algebraic structure. Finally, the behaviour of the 'original' HCF of locally homogeneous non-Kähler metrics on compact complex surfaces, together with a convergence result, was investigated in [13.

Also the pluriclosed flow has been widely studied in the homogeneous case. In [4] it was proved that the flow of left-invariant metrics on 2-step nilpotent Lie groups is immortal (see also [21]); while, in [2] convergence results for normalized solutions to the flow on almost abelian and 2-step nilpotent Lie groups were proved.

In [5], Fei and Phong showed that there exists a relation between the $\mathrm{HCF}_{+}$and the Anomaly flow, which is a metric flow introduced by Phong, Picard and Zhang in [15] to study the Hull-Strominger system (see also 14, 16, 17]). Namely, Fei and Phong proved that any solution to the HCF + starting from a conformally balanced metric $\omega$ on $X$, i.e. a metric satisfying $d\left(\|\Psi\|_{\omega}^{2} \omega^{n-1}\right)=0$ for a complex volume form $\Psi$ on $X$ with $\operatorname{dim}_{\mathbb{C}} X=n$, gives rise to a solution to the Anomaly flow after a time rescaling. 
Thus, since any left-invariant Hermitian metric on a complex unimodular Lie group is balanced [1, our results apply to the Anomaly flow when $\Psi$ is a left-invariant volume form.

Corollary C. Let $N$ be a complex 2-step nilpotent Lie group and $\Psi$ a left-invariant volume form on $N$. Any left-invariant solution $\omega_{t}$ on $N$ to the Anomaly flow

$$
\partial_{t}\left(\|\Psi\|_{\omega_{t}} \omega_{t}^{n-1}\right)=i \partial \bar{\partial} \omega_{t}^{n-2}
$$

is immortal. Moreover, $(1+t)^{-1} \omega_{t}$ subconverges as $t \rightarrow \infty$ to a non-flat left-invariant metric $\bar{\omega}$ on $\bar{N}$ satisfying (3), in the Cheeger-Gromov topology.

In [18] Phong, Picard and Zhang studied the Anomaly flow on complex 3-dimensional unimodular Lie groups. We mention that our corollary slightly extend the long-time existence result obtained in [18] for the complex Heisenberg Lie group. Finally, in [20] the author and Ugarte investigated the behaviour of the Anomaly flow on 2-step nilpotent Lie groups providing the first examples of the Anomaly flow with non-flat holomorphic bundle.

The paper is organized as follows. In Section 1 we explicitly compute the components of the $\mathrm{HCF}_{+-}$ tensor in terms of the Lie bracket. We also recall the bracket flow technique. Section 2 is devoted to the proof of Theorem $\mathrm{A}$. Finally, in Section 3 we prove Theorem B and we give an explicit example.

Notation and conventions. All over the paper we adopt the Einstein convention for the sum over repeated indices. By a complex Lie group we mean a Lie group endowed with a bi-invariant complex structure (that is, the multiplication is a holomorphic map).

Acknowledgments. The author warmly thanks Luigi Vezzoni and Ramiro Lafuente for their interest and helpful comments.

\section{Preliminaries}

1.1. The $\mathbf{H C F}_{+}$on Lie groups. Let $(G, J)$ be a Lie group equipped with a left-invariant complex structure. Let $\mathfrak{g}$ be the Lie algebra of $G$ and let $\mu$ be the Lie bracket on $\mathfrak{g}$. In the following, we compute the tensor $K$ of a left-invariant Hermitian metric $g$ on $G$ in terms of structure constants of $\mathfrak{g}$.

The Chern connection $\nabla$ of $g$ is by definition the unique Hermitian connection $(\nabla g=\nabla J=0)$ with vanishing $(1,1)$-part of the torsion. Therefore, given a left-invariant $g$-unitary frame $\left\{Z_{1}, \ldots, Z_{n}\right\}$ on $G$, we have

$$
T_{l \bar{k}}:=\nabla_{l} Z_{\bar{k}}-\nabla_{\bar{k}} Z_{l}-\mu\left(Z_{l}, Z_{\bar{k}}\right)=0
$$

or, in terms of the Christoffel symbols of $\nabla$,

$$
\Gamma_{l \bar{k}}^{\bar{r}}=\mu_{l \bar{k}}^{\bar{r}}, \quad \Gamma_{\bar{k} l}^{r}=\mu_{\bar{k} l}^{r} .
$$

On the other hand, by $\nabla g=\nabla J=0$ we get

$$
g\left(\nabla_{l} Z_{r}, Z_{\bar{j}}\right)=-g\left(Z_{r}, \nabla_{l} Z_{\bar{j}}\right)=-g\left(Z_{r}, \mu\left(Z_{l}, Z_{\bar{j}}\right)\right)=-\mu_{l \bar{j}}^{\bar{r}},
$$

that is

Therefore, we have

$$
\Gamma_{l r}^{j}=-\mu_{l \bar{j}}^{\bar{r}}
$$

$$
\Omega_{k \bar{l} \bar{i} \bar{j}}=g\left(\nabla_{k} \nabla_{\bar{l}} Z_{i}, Z_{\bar{j}}\right)-g\left(\nabla_{\bar{l}} \nabla_{k} Z_{i}, Z_{\bar{j}}\right)-g\left(\nabla_{\mu\left(Z_{k}, Z_{\bar{l}}\right)} Z_{i}, Z_{\bar{j}}\right)
$$

and hence

Finally, since

$$
S_{i \bar{j}}=-\mu_{\bar{k} i}^{r} \mu_{k \bar{j}}^{\bar{r}}+\mu_{k \bar{r}}^{\bar{i}} \mu_{\bar{k} r}^{j}+\mu_{k \bar{k}}^{r} \mu_{r \bar{j}}^{\bar{i}}-\mu_{k \bar{k}}^{\bar{r}} \mu_{\bar{r} i}^{j} .
$$

by means of (4) we have

$$
T_{i p}:=\nabla_{i} Z_{p}-\nabla_{p} Z_{i}-\mu\left(Z_{i}, Z_{p}\right),
$$

$$
T_{i p}^{k}:=-\mu_{i \bar{k}}^{\bar{p}}+\mu_{p \bar{k}}^{\bar{i}}-\mu_{i p}^{k}, \quad T_{i p \bar{r}}=-\mu_{i \bar{r}}^{\bar{p}}+\mu_{p \bar{r}}^{\bar{i}}-\mu_{i p}^{r},
$$

which implies

$$
2 \widetilde{Q}_{i \bar{j}}=T_{\bar{k} \bar{r} i} T_{k r \bar{j}}=\left(-\mu_{\bar{k} i}^{r}+\mu_{\bar{r} i}^{k}-\mu_{\bar{k} \bar{r}}^{\bar{i}}\right)\left(-\mu_{k \bar{j}}^{\bar{r}}+\mu_{r \bar{j}}^{\bar{k}}-\mu_{k r}^{j}\right) .
$$


As a direct consequence we have the following proposition.

Proposition 1.1. Let $(G, g)$ be a complex Lie group equipped with a left-invariant Hermitian metric. Then, with respect to a left-invariant $g$-unitary frame $\left\{Z_{1}, \ldots, Z_{n}\right\}$ on $G$, we have

$$
K(g)\left(Z_{i}, Z_{\bar{j}}\right)=\frac{1}{2} g\left(\mu\left(Z_{\bar{r}}, Z_{\bar{p}}\right), Z_{i}\right) \cdot g\left(\mu\left(Z_{r}, Z_{p}\right), Z_{\bar{j}}\right),
$$

Proof. Since on complex Lie groups any mixed bracket $\mu\left(Z_{l}, Z_{\bar{s}}\right)$ vanishes, the claim follows by the above computations.

1.2. The bracket flow technique. Here we briefly recall the bracket flow technique introduced by Lauret in [7] to study the Ricci flow on nilmanifolds. This technique provides a method to study a prescribed geometric flow via a flow of Lie brackets and it has been extensively used to investigate different metric flows in Hermitian geometry (see e.g. 2], 4, 6, [10, 21]). Under some natural assumptions, the bracket flow technique also applies to a large class of geometric structures on homogeneous spaces [11.

Let $(G, J)$ be a complex $n$-dimensional Lie group and let $\mathfrak{g}$ be its Lie algebra. Then, the Lie bracket $\mu_{0}$ of $\mathfrak{g}$ belongs to the variety of complex Lie algebras

$$
\mathcal{C}=\left\{\mu \in \Lambda^{2} \mathfrak{g}^{*} \otimes \mathfrak{g}: \mu \text { satisfies the Jacobi identity and } \mu(J \cdot, \cdot)=J \mu(\cdot, \cdot)\right\},
$$

which admits the 'natural' action of

$$
\mathrm{Gl}(\mathfrak{g}, J)=\{\varphi \in \mathrm{Gl}(\mathfrak{g}): \varphi \circ J=J \circ \varphi\} \simeq \mathrm{Gl}_{n}(\mathbb{C})
$$

defined by

$$
\varphi \cdot \mu:=\varphi \mu\left(\varphi^{-1} \cdot, \varphi^{-1} \cdot\right) .
$$

Now, let us consider a left-invariant Hermitian metric $g_{0}$ on $G$. By the biholomorphisms invariance of $K$, there exists a unique solution $\left(g_{t}\right)_{t \in I}, 0 \in I \subseteq \mathbb{R}$, to the $\mathrm{HCF}_{+}$(2) consisting entirely of leftinvariant Hermitian metrics. Moreover, in [10, Theorem 1.1] Lauret proved that there exists a smooth curve $\left(\varphi_{t}\right)_{t \in I} \in \operatorname{Gl}(\mathfrak{g}, J)$, with $\varphi_{0}=\operatorname{Id}_{\mathfrak{g}}$, such that

$$
g_{t}(\cdot, \cdot)=g_{0}\left(\varphi_{t} \cdot, \varphi_{t} \cdot\right) \text {, }
$$

and the family of Lie brackets

satisfies the bracket flow equation

$$
\mu_{t}:=\varphi_{t} \cdot \mu_{0}
$$

$$
\frac{d}{d t} \mu_{t}=-\pi\left(\mathrm{K}_{\mu_{t}}\right) \mu_{t}, \quad \mu_{\left.t\right|_{0}}=\mu_{0} .
$$

Here, $\pi: \operatorname{End}(\mathfrak{g}) \rightarrow \operatorname{End}\left(\Lambda^{2} \mathfrak{g}^{*} \otimes \mathfrak{g}\right)$ denotes the representation of the action defined in (5), i.e.

$$
\pi(A) \mu(\cdot, \cdot)=A \mu(\cdot, \cdot)-\mu(A \cdot, \cdot)-\mu(\cdot, A \cdot), \quad A \in \operatorname{End}(\mathfrak{g}) ;
$$

while $\mathrm{K}_{\mu_{t}}: \mathfrak{g} \rightarrow \mathfrak{g}$ is the endomorphism related to the value of $K\left(g_{t}\right)$ at $e \in G$ by

$$
\mathrm{K}_{\mu_{t}}=\varphi_{t} \mathrm{~K}_{g_{t}} \varphi_{t}^{-1}, \quad g_{t}\left(\mathrm{~K}_{g_{t}} \cdot, \cdot\right)=K\left(g_{t}\right)(\cdot, \cdot) .
$$

\section{The $\mathrm{HCF}_{+}$ON COMPLEX 2-STEP NilPotent LiE GRoups}

This section is devoted to the proof of Theorem $\mathrm{A}$ The main feature in the proof will be a geometric invariant theory result, which implies the convergence claim.

Let $(N, J)$ be a complex 2-step nilpotent Lie group equipped with a left-invariant Hermitian metric $g$. Let $\mathfrak{n}$ be the Lie algebra of $N$ and $\mu_{0}$ its Lie bracket. Let also $\mathfrak{z}$ be the center of $\left(\mathfrak{n}, \mu_{0}\right)$ and $\mathfrak{z}^{\perp}$ its $g$-orthogonal complement, that is $\mathfrak{n}=\mathfrak{z}^{\perp} \oplus \mathfrak{z}$. By means of Proposition 1.1. the endomorphism $\mathrm{K}_{g}$ defined in (8) satisfies

or, equivalently,

$$
\mathrm{K}_{g}(X)=0, \quad \text { for every } X \in \mathfrak{z}^{\perp},
$$

$$
\mathrm{K}_{g}=\left[\begin{array}{ll}
0 & 0 \\
0 & *
\end{array}\right] .
$$

with respect to the block representation $\mathfrak{n}=\mathfrak{z}^{\perp} \oplus \mathfrak{z}$. As a direct consequence, we get 
Proposition 2.1. The $\mathrm{HCF}_{+}$starting from a left-invariant Hermitian metrics on $\mathrm{N}$ preserves the splitting $\mathfrak{g}=\mathfrak{z}^{\perp} \oplus \mathfrak{z}$.

Remark 2.2. A generalization of Proposition 2.1 holds for complex $k$-step nilpotent Lie group, with $k \geq 2$. More precisely, let $(H, g)$ be a complex nilpotent Lie group equipped with a left-invariant Hermitian metric. Let also $\mathfrak{h}$ be the Lie algebra of $H, \mu$ its Lie bracket and $\mathfrak{h}^{\prime}=\mu(\mathfrak{h}, \mathfrak{h})$ its commutator. Then, $\mathrm{K}_{g}(X)=0$ holds for every $X \in\left(\mathfrak{h}^{\prime}\right)^{\perp}$, where $\left(\mathfrak{h}^{\prime}\right)^{\perp}$ is the $g$-orthogonal complement of $\mathfrak{h}^{\prime}$.

Now, let us consider the variety of complex 2-step nilpotent Lie algebras

$$
\mathcal{N}:=\{\mu \in \mathcal{C}: \mu(\mu(\cdot, \cdot), \cdot)=0\},
$$

and the group

$$
\operatorname{Gl}(\mathfrak{z}, J):=\{\tilde{\varphi} \in \operatorname{Gl}(\mathfrak{z}): \tilde{\varphi} \circ J=J \circ \tilde{\varphi}\} \subset \operatorname{Gl}(\mathfrak{n}, J),
$$

which can be considered as a subgroup of $\operatorname{Gl}(\mathfrak{n}, J)$ via the embedding map $\tilde{\varphi} \mapsto\left(\begin{array}{cc}\text { Id } & 0 \\ 0 & \tilde{\varphi}\end{array}\right)$. Moreover, let $\mathfrak{g l}(\mathfrak{z}, J)$ be the Lie algebra of $\operatorname{Gl}(\mathfrak{z}, J)$ and

$$
\mathfrak{p}(\mathfrak{z}, J):=\mathfrak{g l}(\mathfrak{z}, J) \cap \mathfrak{p},
$$

where $\mathfrak{p}=\operatorname{sym}(\mathfrak{n},\langle\cdot, \cdot\rangle)$ is the set of symmetric endomorphisms of $\mathfrak{n}$. Here $\langle\cdot, \cdot\rangle$ denotes any inner product induced by $g$ on any tensor product of $\mathfrak{n}$ and its dual. Then, we have the following fundamental lemma.

Lemma 2.3. The mapping

$$
\mathcal{N} \backslash\{0\} \stackrel{\Phi}{\rightarrow} \mathfrak{p}(\mathfrak{z}, J), \quad \mu \mapsto \frac{2}{\|\mu\|^{2}} \mathrm{~K}_{\mu},
$$

is a moment map for the linear $\mathrm{Gl}(\mathfrak{z}, J)$-action on $\mathcal{N} \backslash\{0\}$, in the sense of real geometric invariant theory. That is,

$$
\left\langle\mathrm{K}_{\mu}, E\right\rangle=\frac{1}{2}\langle\pi(E) \mu, \mu\rangle, \quad \text { for all } E \in \mathfrak{p}(\mathfrak{z}, J), \mu \in \mathcal{N} \backslash\{0\},
$$

where $\pi(E) \mu$ is defined as in (7).

Proof. Let $\left\{Z_{i}\right\}$ be a $\langle\cdot, \cdot\rangle$-unitary basis of $\mathfrak{n}$. By means of the 2-step nilpotency, one gets that

$$
\left\langle\mathrm{K}_{\mu}\left(Z_{i}\right), E\left(Z_{\bar{i}}\right)\right\rangle=\frac{1}{2} \mu_{k r}^{i} \mu_{\bar{k} \bar{r}}^{\bar{j}} E_{\bar{i}}^{\bar{j}}, \quad \text { for any } E \in \mathfrak{p}(\mathfrak{z}, J) .
$$

On the other hand,

$$
\left\langle\pi(E) \mu\left(Z_{r}, Z_{k}\right), \mu\left(Z_{\bar{r}}, Z_{\bar{k}}\right)\right\rangle=\left\langle E \mu\left(Z_{r}, Z_{k}\right), \mu\left(Z_{\bar{r}}, Z_{\bar{k}}\right)\right\rangle=E_{i}^{s} \mu_{r k}^{s} \mu_{\bar{r} \bar{k}}^{\bar{i}},
$$

and the claim follows by the symmetry of $E$.

Let us stress that, a similar result have been found for the 'original' HCF on complex unimodular Lie groups, and $\Phi$ is actually the restriction (up to a positive constant) of that moment map found in [6]. Indeed, if we denote by

$$
\mathcal{N} \backslash\{0\} \rightarrow \operatorname{End}(\mathfrak{n}), \quad \mu \mapsto \frac{4}{\|\mu\|^{2}} \mathrm{M}_{\mu},
$$

the moment map associated to the 'original' $\mathrm{HCF}$, which satisfies

$$
\left\langle\mathrm{M}_{\mu}, A\right\rangle=\frac{1}{2}\langle\pi(A) \mu, \mu\rangle, \quad \text { for all } A \in \operatorname{End}(\mathfrak{n}), \mu \in \mathcal{N} \backslash\{0\},
$$

(see [6, Sec. 3]), a direct computation yields $\left\langle\mathrm{M}_{\mu}, E\right\rangle=2\left\langle\mathrm{~K}_{\mu}, E\right\rangle$ for every $E \in \mathfrak{p}(\mathfrak{z}, J)$.

Remark 2.4. By means of [6, Lemma 2.1], it follows that the endomorphism $K_{\mu}: \mathfrak{n} \rightarrow \mathfrak{n}$ is given by

$$
\mathrm{K}_{\mu}=\left[\begin{array}{cc}
0 & 0 \\
0 & 2 \cdot \operatorname{pr}_{\mathfrak{z}}\left(\operatorname{Ric}_{\mu}\right)
\end{array}\right],
$$

where $\operatorname{Ric}_{\mu}$ is the Ricci curvature endomorphism of the $\operatorname{Ricci}$ curvature tensor $\operatorname{Ric}(g)$ and $\operatorname{pr}_{\mathfrak{z}}$ denotes its orthogonal projection to $\operatorname{End}(\mathfrak{z})$.

We are now in a position to prove Theorem $\mathrm{A}$ 
Proof of Theorem $A$. Let $g_{t}$ be the solution to the $\mathrm{HCF}_{+}$starting at $g_{0}$. By the equivalence of the $\mathrm{HCF}_{+}$ and the bracket flow (6), it is enough to prove that $\mu_{t}$ is defined for all $t \in[0,+\infty)$. Therefore, since Lemma 2.3 holds, we have

$$
\frac{d}{d t}\left\|\mu_{t}\right\|^{2}=2\left\langle\frac{d}{d t} \mu_{t}, \mu_{t}\right\rangle=-2\left\langle\pi\left(\mathrm{K}_{\mu_{t}}\right) \mu_{t}, \mu_{t}\right\rangle=-4\left\|\mathrm{~K}_{\mu_{t}}\right\|^{2} \leq 0,
$$

and the claim follows by standard ODE arguments.

Now, let $\nu_{t}:=\mu_{t} /\left\|\mu_{t}\right\|$ be the norm-normalized bracket flow. Then, by [2, Lemma 2.3], $\nu_{t}$ solves the normalized bracket flow equation

$$
\frac{d}{d t} \nu_{t}=-\pi\left(U_{\nu_{t}}+r_{\nu_{t}} \operatorname{Id}_{\mathfrak{n}}\right) \nu_{t}
$$

where $r_{\nu}:=\left\langle\pi\left(\mathrm{K}_{\nu}\right) \nu, \nu\right\rangle=2\left\|\mathrm{~K}_{\nu}\right\|^{2}$. On the other hand, since (10) is a moment map, by means of [3, Lemma 7.2] the normalized bracket flow turns out to be the negative gradient flow (up to a constant and a time reparameterization) of the real-analytic functional

$$
F: \mathcal{N} \backslash\{0\} \rightarrow \mathbb{R}, \quad \nu \mapsto \frac{\left\|\mathrm{K}_{\nu}\right\|^{2}}{\|\nu\|^{4}} .
$$

Moreover, since $\nu_{t}$ exists for all $t \geq 0$ and the space of unitary bracket is compact, there must exist an accumulation point $\bar{\nu}$ of $\nu_{t}$. Then, by Łojasiewicz's theorem on real-analytical gradient flow, $\nu_{t} \rightarrow \bar{\nu}$ as $t \rightarrow \infty$ and

$$
\pi\left(\mathrm{K}_{\bar{\nu}}+r_{\bar{\nu}} \operatorname{Id}_{\mathfrak{n}}\right) \bar{\nu}=0
$$

i.e. $\bar{\nu}$ is a fixed point of (12). Therefore, in view of (7), $\mathrm{K}_{\bar{\nu}}+r_{\bar{\nu}} \operatorname{Id}_{\mathfrak{n}}$ is a derivation of $\mathfrak{n}$ and its corresponding metric $\bar{g}$ is an algebraic $\mathrm{HCF}_{+}$-soliton. A direct computation yields that

$$
\operatorname{sc}(\bar{g})=\operatorname{tr} \mathrm{K}_{\bar{\nu}}=-\frac{1}{2},
$$

and hence the soliton is non-flat.

Finally, arguing in the same fashion as [2, Theorem A], it is not hard to prove that $\|\mu(t)\| \sim t^{-1 / 2}$ as $t \rightarrow \infty$. On the other hand, scaling the metric by a factor $c>0$ is equivalent to scaling the corresponding bracket by a $c^{-1 / 2}$ factor (see $[9, \S 2.1]$ ). Therefore, since the convergence of the brackets yields subconvergence for the corresponding family of left-invariant metrics in the Cheeger-Gromov sense 8 , Theorem $6.20]$, the claim follows.

\section{Algebraic solitons to the $\mathrm{HCF}_{+}$}

Let $(G, g)$ be a complex non-abelian Lie group equipped with a left-invariant metric. Then, $g$ is said to be a static metric to the $\mathrm{HCF}_{+}$if

$$
K(g)=c g,
$$

for some $c \in \mathbb{R}$.

Proposition 3.1. Any left-invariant $H C F_{+}$-static metric $g$ on $G$ is shrinking (i.e. $c>0$ ).

Proof. The proof directly follows by Proposition 1.1, since $n c=\operatorname{tr}_{g} K(g)=\frac{1}{2}\|\mu\|^{2}$.

As a direct consequence, we get the following

Corollary 3.2. If $G$ is nilpotent, then there are no left-invariant $\mathrm{HCF}_{+}$-static metrics on $\mathrm{G}$.

Proof. Let us suppose that there exists a left-invariant metric $g$ on $G$ satisfying (13). Let $\left\{Z_{1}, \ldots, Z_{n}\right\}$ be a $\langle\cdot, \cdot\rangle$-unitary basis of $\mathfrak{g}$. Since $G$ is nilpotent, there exists at least one $Z_{i}$ such that $\left\langle Z_{i}\right\rangle \perp \mu(\mathfrak{g}, \mathfrak{g})$. By means of Proposition 1.1, one gets

$$
K(g)\left(Z_{i}, Z_{\bar{i}}\right)=0
$$

which is not possible by Proposition 3.1] and hence the claim follows.

Remark 3.3. Given a complex Lie algebra $(\mathfrak{g}, J)$, the complex structure $J$ preserves both the center $\mathfrak{z}$ and the commutator $\mu(\mathfrak{g}, \mathfrak{g})$ of $\mathfrak{g}$. This was implicitly used in the proof of Corollary 3.2

Let us now focus on soliton metrics. 
Definition 3.4. A left-invariant metric $g$ on $G$ is said to be an algebraic $\mathrm{HCF}_{+}$-soliton if

$$
K(g)=c g+\frac{1}{2}(g(D \cdot, \cdot)+g(\cdot, D \cdot)),
$$

for some $c \in \mathbb{R}$ and $D \in \operatorname{Der}(\mathfrak{g})$.

We are now in a position to prove Theorem B

Proof of Theorem $B$. Let $(N, g)$ be a complex (non-abelian) 2-step nilpotent Lie group equipped with an algebraic $\mathrm{HCF}_{+}$-soliton metric. Moreover, let $\mathfrak{n}$ be the Lie algebra of $N, \mu$ its Lie bracket and let us consider the $\langle\cdot, \cdot\rangle$-orthogonal splitting $\mathfrak{n}=\mathfrak{z}^{\perp} \oplus \mathfrak{z}$, where $\mathfrak{z}$ is center of $\mathfrak{n}$. Then, by means of (8), the algebraic soliton condition can be written in terms of $\mathrm{K}_{\mu}$ as

$$
\mathrm{K}_{\mu}=c \operatorname{Id}_{\mathfrak{n}}+\frac{1}{2}\left(D+D^{t}\right),
$$

where $(\cdot)^{t}$ denotes the transpose with respect to the inner product $\langle\cdot, \cdot\rangle$ on $\mathfrak{n}$.

We first show that any derivation $D$ in (14) is a symmetric operator, for which its enough to prove that $D^{t}$ is also a derivation. Since $\mathfrak{n}$ is a complex 2-step nilpotent Lie algebra, it follows that

$$
D=\left[\begin{array}{ll}
* & 0 \\
* & *
\end{array}\right]
$$

where the blocks are in terms of $\mathfrak{n}=\mathfrak{z}^{\perp} \oplus \mathfrak{z}$. On the other hand, by means of (9) and (14), we have

$$
D=\left[\begin{array}{cc}
-c \operatorname{Id}_{\mathfrak{z}^{\perp}} & 0 \\
0 & D_{\mathfrak{z}}
\end{array}\right] \text {. }
$$

Therefore, since the representation $\pi$ is a Lie algebra morphism which satisfies $\pi\left(A^{t}\right)=\pi(A)^{t}$ and Lemma 2.3 holds, we get

$$
2 \operatorname{tr} \mathrm{K}_{\mu}\left[D, D^{t}\right]=\left\langle\pi\left(\left[D, D^{t}\right]\right) \mu, \mu\right\rangle=\left\langle\left[\pi(D), \pi\left(D^{t}\right)\right] \mu, \mu\right\rangle=\left\|\pi\left(D^{t}\right) \mu\right\|^{2} ;
$$

while, equation (14) implies

$$
\operatorname{tr} \mathrm{K}_{\mu}\left[D, D^{t}\right]=c \operatorname{tr}\left[D, D^{t}\right]+\frac{1}{2} \operatorname{tr} D\left[D, D^{t}\right]+\frac{1}{2} \operatorname{tr} D^{t}\left[D, D^{t}\right]=0,
$$

and the claim follows by $\left\|\pi\left(D^{t}\right) \mu\right\|^{2}=0$, i.e. $D^{t} \in \operatorname{Der}(\mathfrak{n})$.

We are now in a position to prove that any algebraic $\mathrm{HCF}_{+}$-soliton on $N$ is expanding (i.e. $c<0$ ). Let us focus on soliton metrics satisfying (14). In view of Corollary 3.2, we already know that $D \neq 0$. Moreover, by means of Theorem $\mathrm{A}$, a soliton metric cannot be shrinking (i.e. $c>0$ ), since it would give rise to a self-similar solution to the $\mathrm{HCF}_{+}$with a finite-time singularity (see e.g. [10]). Thus, we get $c \leq 0$ in (14). On the other hand, if we assume $c=0$, it directly follows by (15) that

$$
\operatorname{tr} D_{\mathfrak{z}}^{2}=\operatorname{tr} \mathrm{K}_{\mu} D_{\mathfrak{z}}=\left\langle\pi\left(D_{\mathfrak{z}}\right) \mu, \mu\right\rangle=0,
$$

which is in contradiction with our hypothesis $D \neq 0$. Hence, every algebraic $\mathrm{HCF}_{+}$-soliton on $N$ has to be expanding.

To finish the proof, we now prove the uniqueness claim. By the proof of Theorem $\mathrm{A}$, the operator $\mathrm{K}_{\mu}$ gives rise to an algebraic $\mathrm{HCF}_{+}$-soliton if and only if it is a critical point of the functional $F(\mu)=$ $\left\|\mathrm{K}_{\mu}\right\|^{2} /\|\mu\|^{4}$. On the other hand, it has been proved in [3, Corollary 9.4] that critical points for the norm of the moment map which lie in a fixed orbit $\operatorname{Gl}(\mathfrak{z}, J) \cdot \mu$ must actually lie in the same $(\mathfrak{p}(\mathfrak{z}, J) \cdot \mu)$-orbit. Then, since two brackets in the same $\mathfrak{p}(\mathfrak{z}, J)$-orbit correspond to isometric left-invariant metrics on $N$, this conclude the proof.

As a direct consequence of the proof of Theorem B, one gets the following corollary.

Corollary 3.5. Let $g$ be an algebraic $\mathrm{HCF}_{+}$-soliton on $\mathrm{N}$. Then, the $\mathrm{HCF}_{+}$-tensor satisfies

$$
K(g)=c g+g(D \cdot, \cdot),
$$

for some $c<0$ and $D \in \operatorname{Der}(\mathfrak{n})$. 
3.1. An explicit example. Let $\mathbb{H}_{3}(\mathbb{C})$ be the complex 3-dimensional Heisenberg Lie group, which can be defined as the matrix group

$$
\mathbb{H}_{3}(\mathbb{C})=\left\{\left(\begin{array}{ccc}
1 & z_{1} & z_{2} \\
0 & 1 & z_{3} \\
0 & 0 & 1
\end{array}\right): z_{i} \in \mathbb{C}\right\} .
$$

This group is 2-step nilpotent and admits a left-invariant (1,0)-frame $\left\{Z_{1}, Z_{2}, Z_{3}\right\}$ such that

$$
\mu\left(Z_{1}, Z_{2}\right)=Z_{3} \text {. }
$$

Proposition 3.6. Every left-invariant Hermitian metric on $\mathbb{H}_{3}(\mathbb{C})$ is an expanding algebraic $H C F_{+-}$ soliton.

Proof. Let $g$ be a left-invariant Hermitian metric on $\mathbb{H}_{3}(\mathbb{C})$. Then, there exists a $g$-unitary $(1,0)$-frame $\left\{W_{1}, W_{2}, W_{3}\right\}$ such that $\mu$ satisfies

$$
\mu\left(W_{1}, W_{2}\right)=s W_{3}, \quad \text { for some } s \in \mathbb{C} .
$$

With respect to this new frame, we have

$$
K_{g}=\frac{1}{2}\left(\begin{array}{ccc}
0 & 0 & 0 \\
0 & 0 & 0 \\
0 & 0 & |s|^{2}
\end{array}\right)
$$

If we set $D:=K_{g}-c I$, then

$$
D W_{1}=D_{11} W_{1}, \quad D W_{2}=D_{22} W_{2}, \quad D W_{3}=D_{33} W_{3},
$$

for some $D_{i}^{i} \in \mathbb{R}$, and hence $D$ is a derivation if and only if

$$
D \mu\left(W_{1}, W_{2}\right)-\mu\left(D W_{1}, W_{2}\right)-\mu\left(W_{1}, D W_{2}\right)=\left(D_{33}-D_{11}-D_{22}\right) W_{3}=0 .
$$

Finally, setting

the claim follows.

$$
c=-\frac{1}{2}|s|^{2}
$$

\section{REFERENCES}

[1] E. Abbena and A. Grassi. Hermitian Left Invariant Metrics on Complex Lie Groups and Cosymplectic Hermitian Manifolds . Boll. Un. Mat. Ital. A, 6:371-379, 1986.

[2] R. Arroyo and R. Lafuente. The longâĂ ̌̌time behavior of the homogeneous pluriclosed flow. Proc. London Math. Soc., 119(1):266-289, 2019.

[3] C. Böhm and R. Lafuente. Real geometric invariant theory. arXiv e-prints, 2017. arXiv:1701.00643.

[4] N. Enrietti, A. Fino, and L. Vezzoni. The pluriclosed flow on nilmanifolds and tamed symplectic forms. J. Geom. Anal., 25(2):883-909, 2015.

[5] T. Fei and D. Phong. Unification of the Kähler-Ricci and Anomaly flows. arXiv e-prints, 2019. arXiv:1905.02274.

[6] R. Lafuente, M. Pujia, and L. Vezzoni. Hermitian curvature flow on unimodular Lie groups and static invariant metrics. Trans. Amer. Math. Soc., 373(6):3967-3993, 2020.

[7] J. Lauret. Ricci soliton homogeneous nilmanifolds. Math. Ann., 319:715-733, 2001.

[8] J. Lauret. Convergence of homogeneous manifolds. J. London Math. Soc., 86(3):701-727, 2012.

[9] J. Lauret. Ricci flow of homogeneous manifolds. Math. Z., 274:373-403, 2013.

[10] J. Lauret. Curvature flows for almost-Hermitian Lie groups. Trans. Amer. Math. Soc., 367:7453-7480, 2015.

[11] J. Lauret. Geometric flows and their solitons on homogeneous spaces. Rend. Semin. Mat. Univ. Politec. Torino, 74(1):55-93, 2016.

[12] F. Panelli and F. Podestà. Hermitian Curvature Flow on compact homogeneous spaces. J. Geom. Anal., DOI: 10.1007/s12220-019-00239-7, 2019.

[13] F. Pediconi and M. Pujia. Hermitian curvature flow on complex locally homogeneous surfaces. Ann. Mat. Pura Appl., DOI: 10.1007/s10231-020-01015-z, 2020.

[14] D. Phong, S. Picard, and X. Zhang. Anomaly flows. Comm. Anal. Geom., 26(4):955-1008, 2018.

[15] D. Phong, S. Picard, and X. Zhang. Geometric flows and Strominger systems. Math. Z., 288(1-2):101-113, 2018.

[16] D. Phong, S. Picard, and X. Zhang. The Anomaly flow and the Fu-Yau equation. Ann. PDE, 4:13, 2018.

[17] D. Phong, S. Picard, and X. Zhang. A flow of conformally balanced metrics with Kähler fixed points. Math. Ann., 374(3-4):2005-2040, 2019.

[18] D. Phong, S. Picard, and X. Zhang. The Anomaly flow on unimodular Lie groups. Contemp. Math., 735:217-237, 2019. 
[19] M. Pujia. Expanding solitons to the Hermitian curvature flow on complex Lie groups. Diff. Geom. Appl., 64:201-216, 2019.

[20] M. Pujia and L. Ugarte. The Anomaly flow on nilmanifolds. arXiv e-prints, 2020. arXiv:2004.06744.

[21] M. Pujia and L. Vezzoni. A remark on the Bismut-Ricci form on 2-step nilmanifolds. C. R. Acad. Sci. Paris, Ser. I, 356:222-226, 2018.

[22] J. Stanfield. The Hermitian Curvature Flow on almost-abelian complex Lie groups. In preparation, 2020.

[23] J. Streets. Pluriclosed flow, Born-Infeld geometry, and rigidity results for generalized Kähler manifold. Comm. Partial Differential Equations, 41(2):318-374, 2016.

[24] J. Streets. Pluriclosed flow on generalized Kähler manifolds with split tangent bundle. J. Reine Angew. Math., 739:241276,2018 .

[25] J. Streets and G. Tian. A parabolic flow of pluriclosed metrics. Int. Math. Res. Not., 16:3101-3133, 2010.

[26] J. Streets and G. Tian. Hermitian curvature flow. J. Eur. Math. Soc, 13(601-634), 2011.

[27] J. Streets and G. Tian. Generalized Kähler geometry and the pluriclosed flow. Nuclear Phys. B, 858(2):366-376, 2012.

[28] J. Streets and G. Tian. Regularity results for pluriclosed flow. Geom. Top., 17(4):2389-2429, 2013.

[29] Y. Ustinovskiy. Hermitian curvature flow on complex homogeneous manifolds. Ann. Scuola Norm. Sup. Pisa Cl. Sci., DOI: 10.2422/2036-2145.201903_011, 2019.

[30] Y. Ustinovskiy. The Hermitian curvature flow on manifolds with non-negative Griffiths curvature. Amer. J. Math., 141(6):1751-1775, 2019.

Dipartimento di Matematica G. Peano, Università di Torino, Via Carlo Alberto 10, 10123 Torino, Italy

E-mail address: mattia.pujia@unito.it 